Draft Version May 12, 2020

Typeset using LATEX twocolumn style in AASTeX63

\title{
Constraining Black Hole Populations in Globular Clusters using Microlensing: Application to Omega Centauri
}

\author{
John Zaris, ${ }^{1}$ DoĞa Veske, ${ }^{1}$ Johan Samsing, ${ }^{2}$ Zsuzsa Márka,${ }^{3}$ Imre Bartos, ${ }^{4}$ And Szabolcs Márka ${ }^{1}$ \\ ${ }^{1}$ Department of Physics, Columbia University in the City of New York, 550 W 120th St., New York, NY 10027, USA \\ ${ }^{2}$ Niels Bohr International Academy, The Niels Bohr Institute, Blegdamsvej 17, DK-2100, Copenhagen, Denmark \\ ${ }^{3}$ Columbia Astrophysics Laboratory, Columbia University in the City of New York, 550 W 120th St., New York, NY 10027, USA \\ ${ }^{4}$ Department of Physics, University of Florida, PO Box 118440, Gainesville, FL 32611-8440, USA
}

\begin{abstract}
We estimate the rate of gravitational microlensing events of cluster stars due to black holes (BHs) in the globular cluster NGC $5139(\omega C e n)$. Theory and observations both indicate that $\omega C e n$ may contain thousands of $\mathrm{BHs}$, but their mass spectrum and exact distribution are not well constrained. In this Letter we show that one may observe microlensing events on a timescale of years in $\omega C e n$, and such an event sample can be used to infer the BH distribution. Direct detection of BHs will, in the near future, play a major role in distinguishing binary BH merger channels. Here we explore how gravitational microlensing can be used to put constraints on $\mathrm{BH}$ populations in globular clusters.
\end{abstract}

Keywords: Black holes (162) — Globular star clusters (656) — Gravitational microlensing (672)

\section{INTRODUCTION}

The detection of gravitational waves (GWs) by Advanced LIGO (Aasi et al. 2015) and Advanced Virgo (Acernese et al. 2014) has confirmed the existence of merging binary black holes (BBHs) (Abbott et al. 2016a,b,c, 2017a,b; Zackay et al. 2019; Venumadhav et al. 2019). However, there is limited evidence to explain how and where this observed $\mathrm{BBH}$ population forms in our universe. The growing list of proposed formation channels includes field binaries (Dominik et al. 2012; Belczynski et al. 2016; Silsbee \& Tremaine 2017; Murguia-Berthier et al. 2017; Rodriguez \& Antonini 2018; Schrøder et al. 2018; Spera et al. 2019; Giacobbo \& Mapelli 2018; Mapelli et al. 2017), active galactic nuclei discs (Bartos et al. 2017; Stone et al. 2017; McKernan et al. 2017; Yang et al. 2019), galactic nuclei (O'Leary et al. 2009; Hong \& Lee 2015; VanLandingham et al. 2016; Antonini \& Rasio 2016; Stephan et al. 2016; Hamers et al. 2018; Fragione et al. 2019), and dynamical assembly in globular clusters (GCs) (Portegies Zwart \& McMillan 2000; Banerjee et al. 2010; Rodriguez et al. 2016a; Askar et al. 2017; Park et al. 2017; Antonini \& Gieles 2019; Ziosi et al. 2014; Mapelli 2016; Di Carlo

Corresponding author: John Zaris

jcz2114@columbia.edu et al. 2019; Fragione \& Kocsis 2018). In this work we study methods to constrain the BH population in GCs independently of GW observations.

Recently, BH candidates have been detected in GCs using a variety of methods, including analysis of X-ray and radio emissions (Strader et al. 2012; Chomiuk et al. 2013; Miller-Jones et al. 2015) and radial velocity measurements of $\mathrm{BH}$ companion stars in binary systems (Giesers et al. 2018, 2019). Stellar-mass BH candidates have even been found in GCs outside of the Milky Way by analyzing X-ray emission patterns (Maccarone et al. 2007, 2011; Shih et al. 2010; Brassington et al. 2010).

Theory and observations indicate that individual GCs are able to retain a large fraction of their initial $\mathrm{BH}$ population, depending on their mass and dynamical history (e.g. Morscher et al. 2015; Rodriguez et al. 2016a; Askar et al. 2018; Kremer et al. 2018; Zocchi et al. 2019; Weatherford et al. 2019). One way of probing this population is through GW observations; but distinguishing BBHs mergers assembled in GCs from those formed through other channels has been shown to be difficult. Using inferred distributions of BH spins (e.g. Rodriguez et al. 2016b), masses (e.g. Zevin et al. 2017), and orbital eccentricities (e.g. Gültekin et al. 2006; Samsing et al. 2014; Samsing \& Ramirez-Ruiz 2017; Samsing \& Ilan 2018; Samsing et al. 2018b; Samsing \& Ilan 2019; Samsing 2018; Samsing et al. 2018a; Zevin et al. 2019; Samsing et al. 2019; Romero-Shaw et al. 2019) from GW 
observations is possible; but gives only an indirect and statistical measure of the contribution of GC BBHs to the set of observed BBH mergers.

In this Letter, we explore the possibility of directly constraining the $\mathrm{BH}$ population of GCs located in the Milky Way (MW) through their gravitational lensing effects (e.g. Udalski et al. 1994; Paczynski 1994; Bennett et al. 2002). If BHs populate the core of GCs, then they will occasionally gravitationally lens and magnify the background cluster stars, an effect known as microlensing (e.g. Paczynski 1986).

Previous microlensing studies have investigated several types of lens-source systems. For example, research has been conducted on the lensing of galactic center stars by GC stars (Paczynski 1994; Pietrukowicz et al. 2012), planetary mass objects (Sahu et al. 2001) and dark matter (Jetzer et al. 1998), and the lensing of GC stars by galactic compact dark matter (Rhoads \& Malhotra 1998) and intermediate-mass BHs theorized to inhabit GCs (Safonova \& Stalin 2010).

In this work, we study the microlensing of GC stars by stellar-mass GC BHs, focusing our attention on the massive GC $\omega C$ Cen. Recent studies indicate that a $\mathrm{BH}$ population with total mass $\sim 10^{5} M_{\odot}$ is likely to occupy the core of $\omega$ Cen (Zocchi et al. 2019), which makes this cluster a particularly interesting candidate to monitor in current and future surveys. Using both analytical and numerical techniques we illustrate that an observable microlensing rate $\sim 1 y^{-1}$ is expected for $\omega C e n$ and investigate how this rate depends on the properties of the BH population. Any detection or non-detection can therefore be used constrain the current $\mathrm{BH}$ distribution in $\omega C$ en. This, in turn, can help determine the degree to which GCs contribute to the observed $\mathrm{BBH}$ merger rate.

The Letter is structured as follows. We begin in Section 2 by applying microlensing theory to the case of a GC, from which we derive an order-of-magnitude estimate for the lensing rate in $\omega C$ Cn. In Section 3 we improve on our rate estimate using a more sophisticated Monte-Carlo (MC) technique, where we take into account the observed stellar profile of $\omega C e n$. We conclude our study in Section 4.

\section{LENSING THEORY AND TOY MODEL}

Here, we first review the standard lensing equations (e.g. Paczynski 1986), which we then use to derive an approximate but closed form expression for the rate of stellar microlensing by BHs in GCs. This expression provides general insight into how the microlensing rate depends on properties such as the mass and velocity dispersion of both the $\mathrm{BH}$ and star distributions.

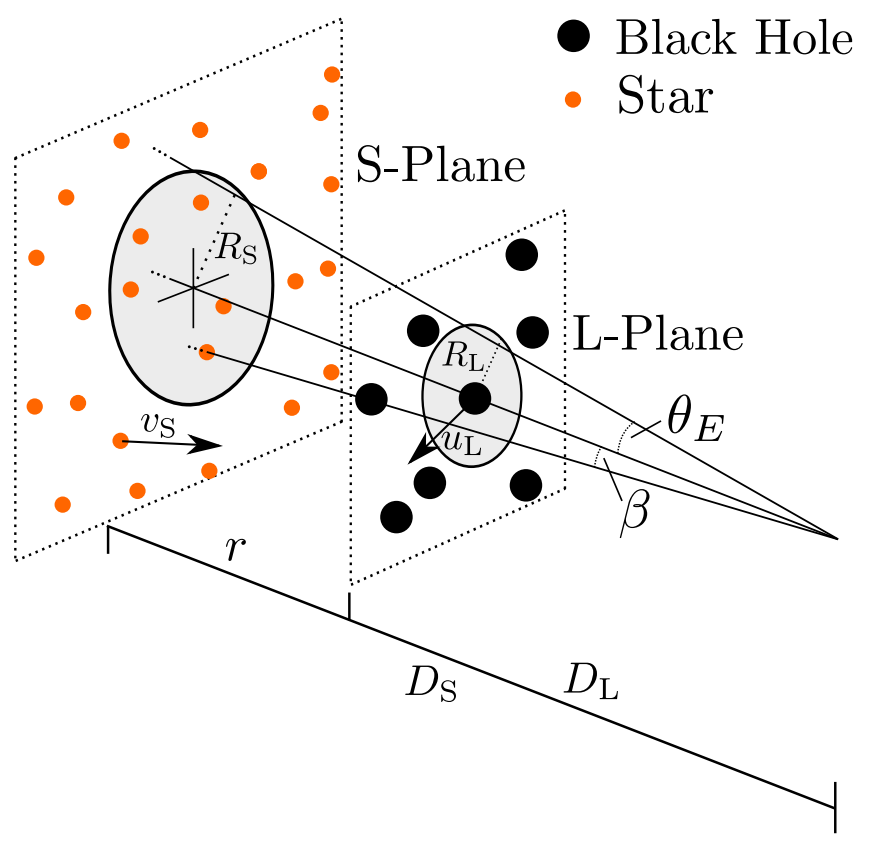

Figure 1. Illustration of the lensing setup described in Section 2. The illustration shows two planes; the source plane (S-Plane), which here is populated with stars (orange dots), and the lensing plane (L-Plane), which is populated with BHs (black dots). The observer is located to the right at a distance $D_{\mathrm{L}}$ and $D_{\mathrm{S}}$ to the L-Plane and S-Plane, respectively. The rate at which stars in the S-Plane cross the Einstein ring (grey circle in the $\mathrm{S}-\mathrm{Plane}$ with radius $R_{\mathrm{S}}$ ) is linked to the observable microlensing rate, as further described in Section 2 .

When a lensing object (the $\mathrm{BH}$ ) passes near the line of sight (l.o.s) from an observer to a source (the star), the source will appear magnified in the observer's frame by a factor

$$
\mu=\frac{\alpha^{2}+2}{\alpha\left(\alpha^{2}+4\right)^{1 / 2}}
$$

where $\alpha$ is the rescaled angular impact parameter defined by

$$
\alpha=\beta / \theta_{E}
$$

In this equation, $\beta$ is the angular distance between the lensed star and the $\mathrm{BH}$, and $\theta_{E}$ is the angular Einstein radius defined by

$$
\theta_{E}=\sqrt{\frac{4 G m_{\mathrm{BH}}}{c^{2}} \frac{D_{\mathrm{S}}-D_{\mathrm{L}}}{D_{\mathrm{S}} D_{\mathrm{L}}}}
$$

where $D_{\mathrm{S}}$ and $D_{\mathrm{L}}$ are the distances from the observer to the lensed star and to the $\mathrm{BH}$, respectively, $m_{\mathrm{BH}}$ is the mass of the lensing BH, $G$ is Newton's gravitational constant, and $c$ is the speed of light. Figure 1 illustrates this setup.

Using these equations, we now derive an expression for the rate of microlensing in a GC consisting of stars and 
BHs. We begin by calculating the rate at which stars located in the source-plane (S-plane) cross the Einstein ring of a given $\mathrm{BH}$ located in the lens-plane (L-plane), where the radii of the Einstein rings in the L-plane and the S-plane are given by $R_{\mathrm{L}} \approx D_{\mathrm{L}} \theta_{E}$ and $R_{\mathrm{S}} \approx D_{\mathrm{S}} \theta_{E}$, respectively. Defining $r$ as the distance between the two planes, it follows that the rate at which stars in the Splane from $r$ to $r+d r$ pass through the Einstein ring is given by

$$
d \Gamma \approx 2 n(r) R_{\mathrm{S}} w d r
$$

where $n(r)$ is the density of stars in the S-plane at distance $r$, and $w$ is the velocity dispersion of the stars relative to the Einstein ring in the S-plane. Note here that we have ignored the curvature of the S-plane, which is a valid approximation as $\theta_{E} \ll 1$. The relative velocity dispersion can be expressed as $w^{2}=v_{\mathrm{S}}^{2}+\left(D_{\mathrm{S}} / D_{\mathrm{L}}\right)^{2} u_{\mathrm{L}}^{2}$, where $v_{\mathrm{S}}$ is the velocity dispersion of the stars in the Splane and $u_{\mathrm{L}}$ is the velocity dispersion of the lensing $\mathrm{BHs}$ in the L-plane. Since $D_{\mathrm{L}} \gg r$ we have that $R_{\mathrm{S}} \approx R_{\mathrm{L}}$, and $w^{2} \approx v_{\mathrm{S}}^{2}+u_{\mathrm{L}}^{2} \approx u_{\mathrm{L}}^{2}$, where the last approximation is accurate within a factor of unity depending on the stellar velocity profile, and on the degree to which the BHs are in energy equipartition with the stars (e.g. Kocsis et al. 2006; Trenti \& van der Marel 2013). Generally, the BHs are located near the center of the GC as they are individually much heavier than the stars. Therefore, their velocity dispersion is $u_{\mathrm{L}} \approx v_{0}$, where $v_{0}$ is the central value. With these approximations, the differential microlensing rate per $\mathrm{BH}$ lens can be written as,

$$
d \Gamma \approx 2 n(r) D_{\mathrm{L}} \theta_{E} v_{0} d r
$$

Expressing the Einstein angle as $\theta_{E}=\sqrt{2 \mathscr{R} r / D_{\mathrm{L}}^{2}}$, where $\mathscr{R}$ is the Schwarzschild radius of a $\mathrm{BH}$ with mass $m_{\mathrm{BH}}$, Eq. (5) can also be written as $d \Gamma=\sqrt{8} n(r) \sqrt{\mathscr{R}} \sqrt{r} v_{0} d r$. This is the rate for stars in an infinitesimally thin slab located at a distance $(r, r+d r)$ from the L-plane, assuming one BH. Therefore, the total rate for a GC with $N_{\mathrm{BH}} \mathrm{BHs}$ is given by

$$
\Gamma \approx \sqrt{8} N_{\mathrm{BH}} n_{0} \mathscr{R}^{2} v_{0}\left(\frac{R_{\mathrm{GC}}}{\mathscr{R}}\right)^{3 / 2} \int n^{\prime} \sqrt{r^{\prime}} d r^{\prime}
$$

where $n^{\prime}=n / n_{0}$ is the stellar density scaled by the cluster's central value, $R_{\mathrm{GC}}$ is the radius of the cluster core, $r^{\prime}=r / R_{\mathrm{GC}}$, and we have assumed that the BHs cluster in the center. As seen, in this simple model we find that $\Gamma \propto N_{\mathrm{BH}} m_{\mathrm{BH}}^{1 / 2} \propto\left(N_{\mathrm{BH}} m_{\mathrm{BH}}\right) m_{\mathrm{BH}}^{-1 / 2}$. Therefore, if the number of BHs is kept fixed $\Gamma \propto m_{\mathrm{BH}}^{1 / 2}$, whereas if the total mass of BHs is kept fixed $\Gamma \propto m_{\mathrm{BH}}^{-1 / 2}$.

We can now use Eq. (6) to provide an estimate for the rate of microlensing events in $\omega C e n$. For this we take $N_{\mathrm{BH}}=10^{4}, m_{\mathrm{BH}}=10 M_{\odot}($ Zocchi et al. 2019), $n_{0}=5 \times 10^{4} p c^{-3}$ (Noyola et al. 2008; D'Souza \& Rix 2013), $v_{0}=25 \mathrm{kms}^{-1}$ (Sollima et al. 2009; Noyola et al. 2010), $R_{\mathrm{GC}}=3.25 p c$ (Trager et al. 1995; Harris 2010), the observable threshold of $\mu$ to be $\mu_{\mathrm{obs}}=1.01$ (Bellini et al. 2017), $\int n^{\prime} \sqrt{r^{\prime}} d r^{\prime}=1$ (this integral is $\approx 1$ for most relevant astrophysical profiles), and find $\Gamma \approx 0.2 y^{-1}$. This rate is promising and serves as our motivation for exploring this problem in greater detail. We continue below with a more accurate numerical approach.

\section{LENSING RATE FOR OMEGA CENTAURI}

Having motivated our lensing study of $\omega C$ en in Section 2 using analytical arguments, we now move on to a more accurate model using MC techniques. Below, we first describe our model of the stars and BHs in $\omega C e n$, after which we present our MC approach and corresponding results.

\subsection{Cluster Model}

Studies of stellar kinematics hint that $\omega C e n$ is likely to harbor a population of BHs with a total mass of $\sim$ $10^{5} M_{\odot}$ (Zocchi et al. 2019); however, the BH mass spectrum and distribution are not well constrained. Therefore, to keep our study as model-independent as possible, we adopt the simple 'energy equipartition' model from Kocsis et al. (2006) to describe the radial position and velocity distributions of the $\mathrm{BHs}$, although we note that GCs likely never acquire perfect equipartition (e.g. Trenti \& van der Marel 2013). In addition, we focus on modeling the microlensing rate from a $\mathrm{BH}$ population with a single mass $m_{\mathrm{BH}}$ to isolate the mass dependence on our results. Following Kocsis et al. (2006), the BHs uniformly distribute within a sphere of radius

$$
R_{\mathrm{BH}}=R_{\mathrm{GC}} \sqrt{\langle m\rangle / m_{\mathrm{BH}}}
$$

with a corresponding velocity dispersion of

$$
\sigma_{\mathrm{BH}}=\sigma_{\mathrm{GC}} \sqrt{\langle m\rangle / m_{\mathrm{BH}}}
$$

where $\sigma_{\mathrm{GC}} \approx \sqrt{(3 / 5) G M_{\mathrm{GC}} / R_{\mathrm{GC}}}, M_{\mathrm{GC}}$ and $R_{\mathrm{GC}}$ are the mass and radius of the cluster core, respectively, and $\langle m\rangle$ is the mean mass of the GC objects (stars + BHs).

In contrast to the $\mathrm{BH}$ population, the stellar distribution in $\omega$ Cen is well constrained from observations. In this study we use the inferred stellar density and velocity dispersion profiles from D'Souza \& Rix (2013) and Sollima et al. (2009), respectively. The former work suggests that the total core mass of $\omega C e n$ is $M_{\mathrm{GC}}=5 \times 10^{5} M_{\odot}$.

\subsection{Monte Carlo method}

With the two distribution models for the BHs and stars presented above, we are now in a position to derive 
the expected microlensing rate for $\omega$ Cen. For this, we developed a $\mathrm{MC}$ code that operates in the following way.

We first generate a $\mathrm{BH}$ assuming that it follows a circular orbit around the center of the core. The inclination angle of the orbit with respect to the l.o.s is randomized uniformly while the orbital radius and velocity are chosen according to Eqs. (7) and (8), respectively. Next, we generate a star whose position and velocity are chosen from the observationally inferred radial density and velocity dispersion profiles, as described in Section 3.1. At each timestep in the BH's orbit, we then estimate the microlensing magnification $\mu$ of the star and store its maximum value, $\mu_{\max }$. This entire process is repeated until a representative sample of star and $\mathrm{BH}$ pairs has been simulated. The final rate can then be calculated by counting the total number of microlensing events per unit time with $\mu_{\max }>\mu_{\mathrm{obs}}$, where $\mu_{\mathrm{obs}}$ is the observational threshold. For the total rate calculation, we assume a total of $3 \times 10^{6}$ visible stars in the GC. Our simulation also calculates the duration of the lensing events. Beginning at the maximum brightness magnification, it records the magnification at each subsequent timestep. From this brightness versus time data, we calculate the minimum time required for the magnification to decrease by the value $\mu_{o b s}-1$, which we define as the event duration. We find that events typically last on the order of several weeks.

\subsection{Results}

Microlensing rates for $\omega$ Cen derived using our MC simulations described in the above Sections 3.1 and 3.2 are shown in Fig. 2. The solid and dashed lines show results for when the total number, $N_{\mathrm{BH}}$, and total mass, $M_{\mathrm{BH}}$, of the BHs are held fixed, respectively. To illustrate the dependence of our results on the uncertain scale of the radial distribution of the $\mathrm{BHs}, R_{\mathrm{BH}}$, we further show, in differently shaded lines, results for when $R_{\mathrm{BH}}$ is varied by a factor of 2 from its fiducial value given by Eq. (7). An important parameter is the magnification threshold $\mu_{o b s}$, defined as the minimum value of $\mu$ (see (1)) associated with an observable brightness magnification. As seen in Figure 8 of Bellini et al. (2017), the photometric error is smaller for brighter stars, so the magnification threshold is also smaller for brighter stars. Since we cannot calculate this value for each individual star, we use two different threshold values for the cluster. From Figure 8 of Bellini et al. (2017), a standard error of 0.1 mags is conservative, as almost all stars have standard errors smaller than this. This leads to our conservative threshold of $\mu_{o b s}=1.1$. We also calculate the rate for $\mu_{o b s}=1.01$, equivalent to a standard error of
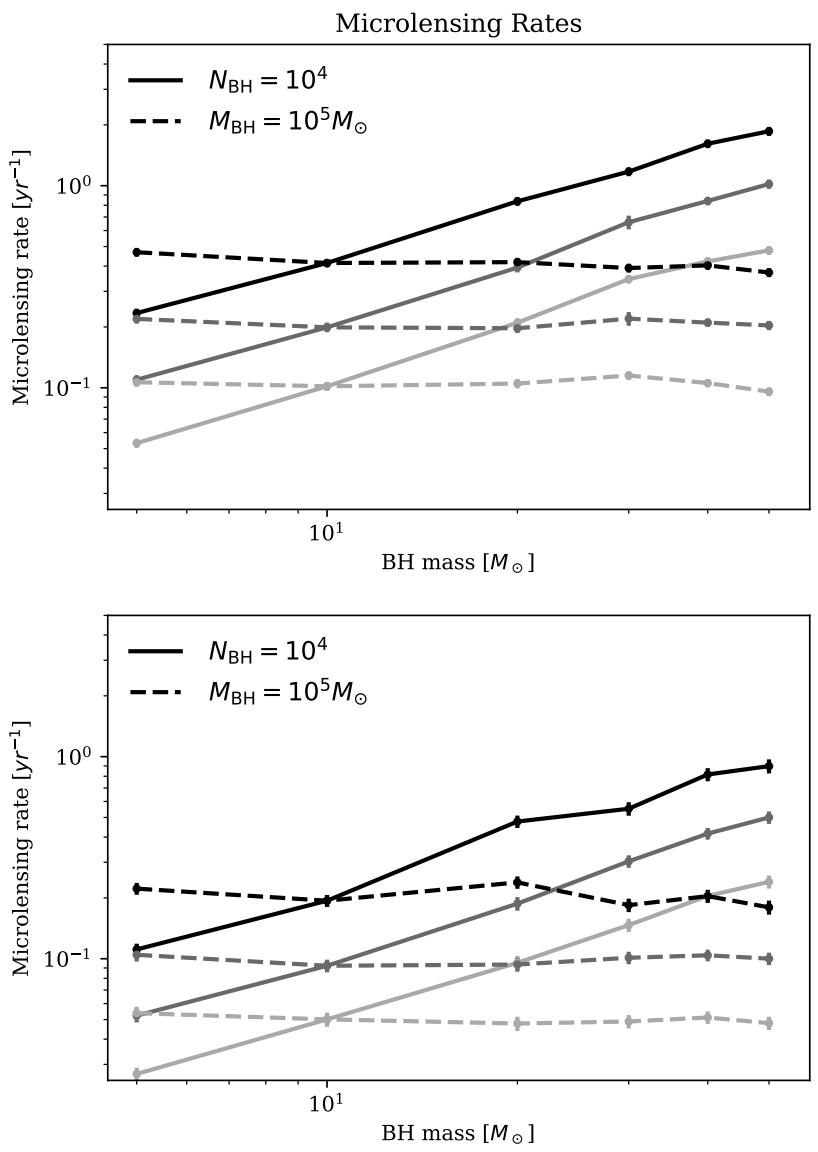

Figure 2. Event rates of cluster stars being microlensed by a corresponding population of cluster BHs in wCen. The rates shown are estimated using $\mathrm{MC}$ techniques as described in Section 3.2, where the stars and BHs are distributed according to the models outlined in Section 3.1. The rates in the top and bottom figures are obtained using magnification thresholds of $\mu_{o b s}=1.01$ and $\mu_{o b s}=1.1$, respectively. The black, dark gray, and light gray lines show results for BH distributions with a radial size of $\{1 / 2,1,2\} \times R_{\mathrm{BH}}$ from Eq. (7), respectively. The solid and dashed lines show results for when the total number, $N_{\mathrm{BH}}$, and total mass, $M_{\mathrm{BH}}$, of the BHs are held fixed at $N_{\mathrm{BH}}=10^{4}$ and $M_{\mathrm{BH}}=10^{5} M_{\odot}$, respectively. Results are discussed in Section 3.3.

0.01 mags. Note that this is approximately the median standard error from Figure 8.

As seen, our numerical results indicate that the expected microlensing rate is in the range $0.1-1 y^{-1}$ for $\omega$ Cen, which is in good agreement with our analytical results from Section 2. For the constant BH number scenario the rate increases slightly faster than $m_{\mathrm{BH}}^{1 / 2}$, and for the constant total $\mathrm{BH}$ mass scenario it decreases slower than $m_{\mathrm{BH}}^{-1 / 2}$. These behaviors can be explained by the localization of the massive BHs closer to the cluster's center where the star density and, consequently, lensing rate are higher. 


\section{DISCUSSION}

Is there a significant population of BHs currently residing in GCs throughout our local volume? That is one of the current major questions in the rising field of GW astrophysics, where merging $\mathrm{BHs}$, but not their origins, are directly observed. As suggested by both theory (e.g. Morscher et al. 2015; Rodriguez et al. 2016a) and observations (e.g. Askar et al. 2018; Kremer et al. 2018; Zocchi et al. 2019; Weatherford et al. 2019), GCs are likely able to retain a non-negligible number of $\mathrm{BHs}$; but direct evidence for $\mathrm{BHs}$ in GCs in the upper mass range observed by Advanced LIGO and Advanced Virgo $\left(\sim 30 M_{\odot}\right)$ is still lacking.

In this Letter we have explored the possibility for constraining $\mathrm{BH}$ populations in GCs through the use of gravitational microlensing. We find it possible to detect BHs in the core of $\omega$ Cen using microlensing observations with an expected rate range of $\sim 0.1-1 \mathrm{yr}^{-1}$. This rate, each individual lensing lightcurve, and the spatial location of the lensed stars all depend on the $\mathrm{BH}$ mass spectrum and distribution. Hence, detections or non-detections of microlensing events can be used to constrain these quantities, although this is not trivial (e.g. Udalski et al. 1994; Paczynski 1994). Furthermore, while we are concerned only with microlensing events in which a $\mathrm{BH}$ acts as the lens for a cluster star, it is also possible for another cluster star to serve as the lens. Observationally, these two cases are distinguishable. A star lens can be observed optically while a BH lensing event is characterized by an unobservable lens.

This strategy described in this Letter is naturally not limited to $\omega C e n$, but can be applied to any of the $\sim 150$ GCs in the MW. However, it is important to keep in mind that $\omega C e n$ is a unique $\mathrm{GC}$ which likely has an unusually high microlensing rate. $\omega$ Cen has a greater mass than other Galactic GCs (e.g. Baumgardt \& Hilker (2018)), and has even been proposed to be a tidally stripped dwarf galaxy (Majewski et al. 2000; Ibata et al. 2019). Additionally, it cannot be assumed that all GCs contain as many BHs as $\omega$ Cen. However, several clusters analyzed in recent works likely contain hundreds of $\mathrm{BHs}$ and, therefore, may have significant microlensing rates (Arca Sedda et al. 2018; Askar et al. 2018; Kremer et al. 2018; Weatherford et al. 2019).

Observing $\mathrm{BH}$ microlesning events in GCs is challenging, but will likely soon become possible as telescopes with improved performance continue to be constructed. This includes both ground-based telescopes, such as the Thirty Meter Telescope, and space-based ones, such as The Wide Field Infrared Survey Telescope (WFIRST). Past studies of data from the Hubble Space Telescope have already been used to analyze microlensing events near the galactic center and have successfully constrained lens masses (e.g. Kains et al. 2017). Determining the mass of GC BHs may be more difficult as the source and lens distances are similar and, therefore, must be measured precisely. Our simulations suggest that microlensing events last on the order of several weeks. Therefore, observing the cluster on the order of once every few days should provide sufficient data to capture most lensing events. Even though resolution continues to improve, observations near the GC core center may still face the issue of crowding, in which it is impossible to resolve two nearby stars. In this case, lensing will still be observable, but since the localization of the lensed stars can be ambiguous, the uncertainty of the inferred $\mathrm{BH}$ population parameters will be higher. In follow up work we will study how to optimize current and future search strategies for observing such $\mathrm{BH}$ microlensing events.

\section{ACKNOWLEDGEMENTS}

The authors thank Noé Kains for discussions. The authors appreciate the generous support of Columbia University in the City of New York and the University of Florida. JZ is grateful for support from the Ng Teng Fong Student Internship Fund administered through the Columbia College Summer Fund. JS acknowledges support from the Lyman Spitzer Fellowship, and funding from the European Unions Horizon 2020 research and innovation programme under the Marie Sklodowska-Curie grant agreement No. 844629. DV is grateful to the Ph.D. grant of the Fulbright foreign student program.

\section{REFERENCES}

Aasi, J., et al. 2015, Classical and Quantum Gravity, 32, 074001, doi: 10.1088/0264-9381/32/7/074001

Abbott, B. P., Abbott, R., Abbott, T. D., et al. 2016a, Physical Review Letters, 116, 061102, doi: 10.1103/PhysRevLett.116.061102

—. 2016b, Physical Review Letters, 116, 241103, doi: 10.1103/PhysRevLett.116.241103
—. 2016c, Physical Review X, 6, 041015, doi: 10.1103/PhysRevX.6.041015

—. 2017a, Physical Review Letters, 118, 221101, doi: 10.1103/PhysRevLett.118.221101

—. 2017b, Physical Review Letters, 119, 141101, doi: 10.1103/PhysRevLett.119.141101 
Acernese, F., et al. 2014, Classical and Quantum Gravity, 32, 024001, doi: 10.1088/0264-9381/32/2/024001

Antonini, F., \& Gieles, M. 2019, arXiv e-prints, arXiv:1906.11855. https://arxiv.org/abs/1906.11855

Antonini, F., \& Rasio, F. A. 2016, ApJ, 831, 187, doi: $10.3847 / 0004-637 \mathrm{X} / 831 / 2 / 187$

Arca Sedda, M., Askar, A., \& Giersz, M. 2018, MNRAS, 479, 4652, doi: 10.1093/mnras/sty 1859

Askar, A., Arca Sedda, M., \& Giersz, M. 2018, MNRAS, 478, 1844, doi: 10.1093/mnras/sty1186

Askar, A., Szkudlarek, M., Gondek-Rosińska, D., Giersz, M., \& Bulik, T. 2017, MNRAS, 464, L36, doi: $10.1093 / \mathrm{mnrasl} / \mathrm{slw} 177$

Banerjee, S., Baumgardt, H., \& Kroupa, P. 2010, MNRAS, 402, 371, doi: 10.1111/j.1365-2966.2009.15880.x

Bartos, I., Kocsis, B., Haiman, Z., \& Márka, S. 2017, ApJ, 835, 165, doi: 10.3847/1538-4357/835/2/165

Baumgardt, H., \& Hilker, M. 2018, MNRAS, 478, 1520, doi: 10.1093/mnras/sty1057

Belczynski, K., Repetto, S., Holz, D. E., et al. 2016, ApJ, 819, 108, doi: 10.3847/0004-637X/819/2/108

Bellini, A., Anderson, J., Bedin, L. R., et al. 2017, ApJ, 842, 6, doi: 10.3847/1538-4357/aa7059

Bennett, D. P., Becker, A. C., Quinn, J. L., et al. 2002, ApJ, 579, 639, doi: 10.1086/342225

Brassington, N. J., Fabbiano, G., Blake, S., et al. 2010, ApJ, 725, 1805, doi: 10.1088/0004-637X/725/2/1805

Chomiuk, L., Strader, J., Maccarone, T. J., et al. 2013, ApJ, 777, 69, doi: 10.1088/0004-637X/777/1/69

Di Carlo, U. N., Giacobbo, N., Mapelli, M., et al. 2019, Monthly Notices of the Royal Astronomical Society, 487, 29472960, doi: 10.1093/mnras/stz1453

Dominik, M., Belczynski, K., Fryer, C., et al. 2012, ApJ, 759, 52, doi: 10.1088/0004-637X/759/1/52

D'Souza, R., \& Rix, H.-W. 2013, MNRAS, 429, 1887, doi: 10.1093/mnras/sts426

Fragione, G., Grishin, E., Leigh, N. W. C., Perets, H. B., \& Perna, R. 2019, MNRAS, 488, 47, doi: 10.1093/mnras/stz1651

Fragione, G., \& Kocsis, B. 2018, PhRvL, 121, 161103, doi: 10.1103/PhysRevLett.121.161103

Giacobbo, N., \& Mapelli, M. 2018, Monthly Notices of the Royal Astronomical Society, 480, 2011, doi: 10.1093/mnras/sty1999

Giesers, B., Dreizler, S., Husser, T.-O., et al. 2018, MNRAS, 475, L15, doi: 10.1093/mnrasl/slx203

Giesers, B., Kamann, S., Dreizler, S., et al. 2019, A\&A, 632, A3, doi: 10.1051/0004-6361/201936203

Gültekin, K., Miller, M. C., \& Hamilton, D. P. 2006, ApJ, 640,156
Hamers, A. S., Bar-Or, B., Petrovich, C., \& Antonini, F. 2018, ApJ, 865, 2, doi: 10.3847/1538-4357/aadae2

Harris, W. E. 2010, arXiv e-prints, arXiv:1012.3224. https://arxiv.org/abs/1012.3224

Hong, J., \& Lee, H. M. 2015, MNRAS, 448, 754, doi: $10.1093 / \mathrm{mnras} / \mathrm{stv} 035$

Ibata, R. A., Bellazzini, M., Malhan, K., Martin, N., \& Bianchini, P. 2019, Nature Astronomy, 3, 667, doi: 10.1038/s41550-019-0751-x

Jetzer, P., Straessle, M., \& Wandeler, U. 1998, A\&A, 336, 411. https://arxiv.org/abs/astro-ph/9807101

Kains, N., Calamida, A., Sahu, K. C., et al. 2017, ApJ, 843, 145, doi: 10.3847/1538-4357/aa78eb

Kocsis, B., Gáspár, M. E., \& Márka, S. 2006, ApJ, 648, 411, doi: 10.1086/505641

Kremer, K., Ye, C. S., Chatterjee, S., Rodriguez, C. L., \& Rasio, F. A. 2018, ApJ, 855, L15, doi: $10.3847 / 2041-8213 /$ aab26c

Maccarone, T. J., Kundu, A., Zepf, S. E., \& Rhode, K. L. 2007, Nature, 445, 183, doi: 10.1038/nature05434

—. 2011, MNRAS, 410, 1655, doi: 10.1111/j.1365-2966.2010.17547.x

Majewski, S. R., Patterson, R. J., Dinescu, D. I., et al. 2000, in Liege International Astrophysical Colloquia, Vol. 35, Liege International Astrophysical Colloquia, ed. A. Noels, P. Magain, D. Caro, E. Jehin, G. Parmentier, \& A. A. Thoul, 619.

https://arxiv.org/abs/astro-ph/9910278

Mapelli, M. 2016, Monthly Notices of the Royal Astronomical Society, 459, 34323446, doi: $10.1093 / \mathrm{mnras} / \mathrm{stw} 869$

Mapelli, M., Giacobbo, N., Ripamonti, E., \& Spera, M. 2017, MNRAS, 472, 2422, doi: 10.1093/mnras/stx2123

McKernan, B., Ford, K. E. S., Bellovary, J., et al. 2017, ArXiv e-prints. https://arxiv.org/abs/1702.07818

Miller-Jones, J. C. A., Strader, J., Heinke, C. O., et al. 2015, MNRAS, 453, 3918, doi: 10.1093/mnras/stv1869

Morscher, M., Pattabiraman, B., Rodriguez, C., Rasio, F. A., \& Umbreit, S. 2015, ApJ, 800, 9, doi: 10.1088/0004-637X/800/1/9

Murguia-Berthier, A., MacLeod, M., Ramirez-Ruiz, E., Antoni, A., \& Macias, P. 2017, ApJ, 845, 173, doi: $10.3847 / 1538-4357 /$ aa 8140

Noyola, E., Gebhardt, K., \& Bergmann, M. 2008, ApJ, 676, 1008, doi: $10.1086 / 529002$

Noyola, E., Gebhardt, K., Kissler-Patig, M., et al. 2010, ApJL, 719, L60, doi: 10.1088/2041-8205/719/1/L60

O'Leary, R. M., Kocsis, B., \& Loeb, A. 2009, MNRAS, 395, 2127, doi: 10.1111/j.1365-2966.2009.14653.x

Paczynski, B. 1986, ApJ, 304, 1, doi: 10.1086/164140 
-. 1994, AcA, 44, 235.

https://arxiv.org/abs/astro-ph/9407096

Park, D., Kim, C., Lee, H. M., Bae, Y.-B., \& Belczynski, K. 2017, MNRAS, 469, 4665, doi: 10.1093/mnras/stx1015

Pietrukowicz, P., Minniti, D., Jetzer, P., Alonso-García, J., \& Udalski, A. 2012, ApJL, 744, L18, doi: 10.1088/2041-8205/744/2/L18

Portegies Zwart, S. F., \& McMillan, S. L. W. 2000, ApJ, 528, L17

Rhoads, J. E., \& Malhotra, S. 1998, ApJL, 495, L55, doi: 10.1086/311211

Rodriguez, C. L., \& Antonini, F. 2018, ApJ, 863, 7, doi: $10.3847 / 1538-4357 /$ aacea4

Rodriguez, C. L., Chatterjee, S., \& Rasio, F. A. 2016a, PhRvD, 93, 084029, doi: 10.1103/PhysRevD.93.084029

Rodriguez, C. L., Zevin, M., Pankow, C., Kalogera, V., \& Rasio, F. A. 2016b, ApJL, 832, L2, doi: $10.3847 / 2041-8205 / 832 / 1 /$ L2

Romero-Shaw, I. M., Lasky, P. D., \& Thrane, E. 2019, arXiv e-prints, arXiv:1909.05466.

https://arxiv.org/abs/1909.05466

Safonova, M., \& Stalin, C. S. 2010, NewA, 15, 450, doi: 10.1016/j.newast.2009.12.004

Sahu, K. C., Casertano, S., Livio, M., et al. 2001, Nature, 411,1022

Samsing, J. 2018, PhRvD, 97, 103014, doi: 10.1103/PhysRevD.97.103014

Samsing, J., Askar, A., \& Giersz, M. 2018a, ApJ, 855, 124, doi: $10.3847 / 1538-4357 /$ aaab52

Samsing, J., D’Orazio, D. J., Kremer, K., Rodriguez, C. L., \& Askar, A. 2019, arXiv e-prints, arXiv:1907.11231. https://arxiv.org/abs/1907.11231

Samsing, J., \& Ilan, T. 2018, MNRAS, 476, 1548, doi: $10.1093 /$ mnras/sty197

—. 2019, MNRAS, 482, 30, doi: 10.1093/mnras/sty2249

Samsing, J., MacLeod, M., \& Ramirez-Ruiz, E. 2014, ApJ, 784, 71, doi: 10.1088/0004-637X/784/1/71

—. 2018b, ApJ, 853, 140, doi: 10.3847/1538-4357/aaa715

Samsing, J., \& Ramirez-Ruiz, E. 2017, ApJL, 840, L14, doi: 10.3847/2041-8213/aa6f0b

Schrøder, S. L., Batta, A., \& Ramirez-Ruiz, E. 2018, ApJL, 862, L3, doi: 10.3847/2041-8213/aacf8d

Shih, I. C., Kundu, A., Maccarone, T. J., Zepf, S. E., \& Joseph, T. D. 2010, ApJ, 721, 323, doi: 10.1088/0004-637X/721/1/323
Silsbee, K., \& Tremaine, S. 2017, ApJ, 836, 39, doi: $10.3847 / 1538-4357 /$ aa5729

Sollima, A., Bellazzini, M., Smart, R. L., et al. 2009, MNRAS, 396, 2183, doi: 10.1111/j.1365-2966.2009.14864.x

Spera, M., Mapelli, M., Giacobbo, N., et al. 2019, Monthly Notices of the Royal Astronomical Society, 485, 889, doi: 10.1093/mnras/stz359

Stephan, A. P., Naoz, S., Ghez, A. M., et al. 2016, MNRAS, 460, 3494, doi: 10.1093/mnras/stw1220

Stone, N. C., Metzger, B. D., \& Haiman, Z. 2017, MNRAS, 464, 946, doi: 10.1093/mnras/stw2260

Strader, J., Chomiuk, L., Maccarone, T. J., Miller-Jones, J. C. A., \& Seth, A. C. 2012, Nature, 490, 71, doi: $10.1038 /$ nature11490

Trager, S. C., King, I. R., \& Djorgovski, S. 1995, AJ, 109, 218, doi: 10.1086/117268

Trenti, M., \& van der Marel, R. 2013, MNRAS, 435, 3272, doi: 10.1093/mnras/stt1521

Udalski, A., Szymanski, M., Stanek, K. Z., et al. 1994, AcA, 44, 165. https://arxiv.org/abs/astro-ph/9407014

VanLandingham, J. H., Miller, M. C., Hamilton, D. P., \& Richardson, D. C. 2016, ApJ, 828, 77, doi: 10.3847/0004-637X/828/2/77

Venumadhav, T., Zackay, B., Roulet, J., Dai, L., \& Zaldarriaga, M. 2019, arXiv e-prints, arXiv:1904.07214. https://arxiv.org/abs/1904.07214

Weatherford, N. C., Chatterjee, S., Kremer, K., \& Rasio, F. A. 2019, arXiv e-prints, arXiv:1911.09125.

https://arxiv.org/abs/1911.09125

Yang, Y., Bartos, I., Gayathri, V., et al. 2019, Phys. Rev. Lett., 123, 181101, doi: 10.1103/PhysRevLett.123.181101

Zackay, B., Venumadhav, T., Dai, L., Roulet, J., \& Zaldarriaga, M. 2019, arXiv e-prints, arXiv:1902.10331. https://arxiv.org/abs/1902.10331

Zevin, M., Pankow, C., Rodriguez, C. L., et al. 2017, ApJ, 846, 82, doi: 10.3847/1538-4357/aa8408

Zevin, M., Samsing, J., Rodriguez, C., Haster, C.-J., \& Ramirez-Ruiz, E. 2019, ApJ, 871, 91, doi: 10.3847/1538-4357/aaf6ec

Ziosi, B. M., Mapelli, M., Branchesi, M., \& Tormen, G. 2014, Monthly Notices of the Royal Astronomical Society, 441, 37033717, doi: 10.1093/mnras/stu824

Zocchi, A., Gieles, M., \& Hénault-Brunet, V. 2019, MNRAS, 482, 4713, doi: 10.1093/mnras/sty1508 